Appl Math Optim 49:113-121 (2004)

DOI: $10.1007 / \mathrm{s} 00245-003-0786-2$

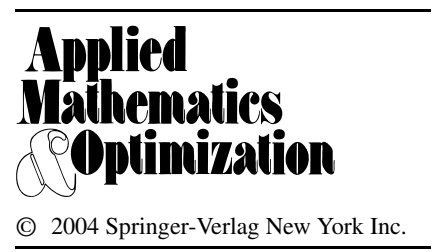

\title{
Stochastic Control for a Class of Random Evolution Models*
}

\author{
Max-Olivier Hongler, ${ }^{1}$ Halil Mete Soner, ${ }^{2}$ and Ludwig Streit ${ }^{3}$ \\ ${ }^{1}$ Institut de Production et Robotique / (LPM) \\ Ecole Polytechnique Fédérale de Lausanne, \\ CH-1015 Lausanne, Switzerland \\ max.hongler@epfl.ch \\ 2 Department of Mathematics, Koc University, \\ Fener Yolu Caddesi, Sariyer 80910, Istanbul, Turkey \\ msoner@ku.edu.tr \\ ${ }^{3}$ CCM-Centro de Ciencias Matematicas, Universidade da Madeira, \\ P-9000 Funchal, Portugal \\ and \\ BiBoS, University of Bielefeld, \\ D-33615 Bielefeld, Germany \\ streit@Physik.Uni-Bielefeld.DE
}

\begin{abstract}
We construct the explicit connection existing between a solvable model of the discrete velocities non-linear Boltzmann equation and the Hamilton-BellmanJacobi equation associated with a simple optimal control of a piecewise deterministic process. This study extends the known relation that exists between the Burgers equation and a simple controlled diffusion problem. In both cases the resulting partial differential equations can be linearized via a logarithmic transformation and hence offer the possibility to solve physically relevant non-linear field models in full generality.
\end{abstract}

Key Words. Piecewise deterministic evolutions, Stochastic optimal control, Logarithmic transformation, Nonlinear field equations.

AMS Classification. 60G40, 93C10.

* This research was partially supported by the Fundaçao para a Ciência e a Tecnologica, Portugal, and the first author was partially supported by the Fonds National Suisse pour la Recherche Scientifique. 


\section{Introduction}

In an influential paper written in 1950, Hopf [Hop] connected the Burgers non-linear field equation and the heat equation by using a logarithmic transformation, i.e. the now famous Hopf-Cole transformation. In several recent contributions, the special role played by this transformation in the domain of stochastic control has been described by Fleming [FL], [FS] and Holland [Hol]. In their work the authors show how certain classes of dynamic programming equations can be linearized by logarithmic transformation. In this context, the Burgers equation can be directly interpreted as the dynamic programming equation of a simple optimal stochastic control problem involving a diffusion process. In the present paper we show that the discrete velocity model of the Boltzmann equation solved by Ruijgrok and $\mathrm{Wu}[\mathrm{RW}]$, is also closely related to the dynamic programming equations (DPE) of a simple control problem involving a piecewise deterministic process. Again a logarithmic transformation enables us to linearize the DPE which explains why exact solutions can be obtained for a non-linear Boltzmann equation. This observation offers a new understanding on the exceptional properties of the Ruijgrok-Wu $(\mathrm{R}-\mathrm{W})$ model and might guide further investigations toward possible generalizations.

Our paper is organized as follows: in Section 2 we briefly recall some basic physical observations concerning the R-W and the Burgers equations. Part of this material has already been presented, in detail, in a previous paper [HS]. In Section 2.1 the connection of the Burgers equation with the optimal control of a simple diffusive process is briefly reviewed. In Section 3, which is the core of this contribution, we construct the stochastic control problem for which the DPE is directly related to the R-W model. Some perspectives and conclusions are given in Section 4.

\section{The Burgers and the Ruijgrok-Wu Model}

A completely solvable model of the non-linear Boltzmann equation has been constructed by Ruijgrok and $\mathrm{Wu}[\mathrm{RW}]$. The R-W model describes particles in collisions in a onedimensional space. These particles are allowed to take only two discrete and opposite velocities, i.e. $\{-\sqrt{D / \alpha}, \sqrt{D / \alpha}\}$. The R-W model can be written as a set of coupled non-linear field equations in the form

$$
\frac{\partial}{\partial t} f(x, t)+\sqrt{\frac{D}{\alpha}} \frac{\partial}{\partial x} f(x, t)=(2 \alpha)^{-1}[2 f(x, t) g(x, t)-f(x, t)+g(x, t)]
$$

and

$$
\frac{\partial}{\partial t} g(x, t)-\sqrt{\frac{D}{\alpha}} \frac{\partial}{\partial x} g(x, t)=-(2 \alpha)^{-1}[2 f(x, t) g(x, t)-f(x, t)+g(x, t)],
$$

where $0<D \quad\left(\mathrm{~m}^{2} \mathrm{~s}^{-1}\right)$ is a diffusion constant, and $0<\alpha \quad$ (s) is a time scale characterizing the collision rate. The functions $f(x, t) \geq 0$ and $g(x, t) \geq 0, x \in \mathbb{R}$, $t \in \mathbb{R}^{+}$, describe the probability density functions to find particles with velocities $\sqrt{D / \alpha}$ (respectively $-\sqrt{D / \alpha}$ ), at position $x$ at time $t$. By introducing the logarithmic 
transformations $[\mathrm{RW}]$

$$
f(x, t)=\left[\alpha \frac{\partial}{\partial t}-\sqrt{D \alpha} \frac{\partial}{\partial x}\right] \ln (\varphi(x, t))
$$

and

$$
g(x, t)=-\left[\alpha \frac{\partial}{\partial t}+\sqrt{D \alpha} \frac{\partial}{\partial x}\right] \ln (\varphi(x, t)),
$$

we observe that (1) can be reduced to

$$
\left[\frac{\partial^{2}}{\partial t^{2}}+\frac{1}{\alpha} \frac{\partial}{\partial t}\right] \varphi(x, t)=\frac{D}{\alpha} \frac{\partial^{2}}{\partial x^{2}} \varphi(x, t) .
$$

The linearity of the field equation (4) directly explains why the R-W model can be solved for any initial conditions. Indeed, given the integrable initial values $f(x, t=0)=f(x)$ and $g(x, t=0)=g(x)$, we can write the explicit form of the solutions $f(x, t)$ and $g(x, t)$. To simplify the expressions, we focus on the case where $\alpha=D=1$ which follows from the original situation by rescaling the variables as $t \mapsto(1 / \alpha) t$ and $x \mapsto$ $(1 / \sqrt{D \alpha}) x$. In these rescaled variables, we can write [RW]

$$
f(x, t)=\left[\frac{\partial}{\partial t}-\frac{\partial}{\partial x}\right] \ln \varphi(x, t)
$$

and

$$
g(x, t)=-\left[\frac{\partial}{\partial t}-\frac{\partial}{\partial x}\right] \ln \varphi(x, t),
$$

with the notation

$$
\begin{aligned}
e^{t / 2} \varphi(x, t)= & \frac{1}{2}[A(x+t)+A(x-t)]+\frac{1}{2} \int_{x-t}^{x+t} d y \mathcal{I}_{0}\left(\frac{1}{2} \sqrt{t^{2}-(x-y)^{2}}\right) B(y) \\
& +\frac{t}{4} \int_{x-t}^{x+t} d y \frac{\mathcal{I}_{1}\left(\frac{1}{2} \sqrt{t^{2}-(x-y)^{2}}\right)}{\sqrt{t^{2}-(x-y)^{2}}} A(y),
\end{aligned}
$$

where $\mathcal{I}_{0}(x)$ and $\mathcal{I}_{1}(x)$ are integer order modified Bessel functions [AS]. Finally, the intial conditions $f(x)$ and $g(x)$ enter into (7) via the $A(x)$ and $B(x)$ functions:

$$
A(x):=\exp \left\{-\frac{1}{2} \int_{-\infty}^{x}[f(y)+g(y)] d y\right\}
$$

and

$$
B(x):=\frac{1}{2}[f(x)-g(x)+1] \exp \left\{-\frac{1}{2} \int_{-\infty}^{x}[f(y)+g(y)] d y\right\} .
$$

The logarithmic transformations given in (2) and (3) suggest that there should be some relation between the R-W model and the Burgers equation which is also linearized by a 
logarithmic transformation, i.e. the Hopf-Cole transformation. Explicitly, recall that the Burgers equation reads

$$
\frac{\partial}{\partial t} h(x, t)=\frac{D}{2} \frac{\partial^{2}}{\partial x^{2}} h(x, t)-2 h(x, t) \frac{\partial}{\partial x} h(x, t),
$$

with $0<D \quad\left(\mathrm{~m}^{2} \mathrm{~s}^{-1}\right)$ and $h(x, t) \geq 0, x \in R, t \in R^{+}$, and the Hopf-Cole transformation [Hop]

$$
h(x, t)=-\frac{D}{2} \frac{\partial}{\partial x} \ln \varphi(x, t)
$$

reduces (8) to

$$
\frac{\partial}{\partial t} \varphi(x, t)=\frac{D}{2} \frac{\partial^{2}}{\partial x^{2}} \varphi(x, t) .
$$

Note that both (10) and (4) are closely related to stochastic processes. Indeed, (10) is directly interpreted as the Chapman-Kolmogorov equation associated with the diffusion process

$$
d X(t)=\sqrt{D} d W_{t},
$$

where $W_{t}$ is the standard Brownian Motion (BM).

On the other hand, (4), which is equivalent to the hyperbolic, linear system

$$
\frac{\partial}{\partial t} \varphi_{a}(x, t)+\sqrt{\frac{D}{\alpha}} \frac{\partial}{\partial x} \varphi_{a}(x, t)=-\frac{1}{2 \alpha}\left[\varphi_{a}(x, t)-\varphi_{b}(x, t)\right]
$$

and

$$
\frac{\partial}{\partial t} \varphi_{b}(x, t)-\sqrt{\frac{D}{\alpha}} \frac{\partial}{\partial x} \varphi_{b}(x, t)=\frac{1}{2 \alpha}\left[\varphi_{a}(x, t)-\varphi_{b}(x, t)\right],
$$

describes the Chapman-Kolmogorov equation characterizing the probability law of the random evolution:

$$
\frac{d}{d t} X(t)=I(t)
$$

where $I(t)$ is an alternating, Markovian, renewal process with the states $\{-\sqrt{D / \alpha}$, $\sqrt{D / \alpha}\}$ and the holding time in the states is a random exponential variable with parameter $\alpha$. The functions $\varphi_{a}(x, t)$ and $\varphi_{b}(x, t)$ describe the probability density having process $X(t)$ at position $x$, at time $t$, with $I(t)$ in the state $-\sqrt{D / \alpha}$ (respectively $+\sqrt{D / \alpha}$ ). Random evolution models of the type given in (13) are studied in detail in [Pi]. From the fact that (4) generalizes (10) and (13) generalizes (11), we conclude, following [HS], that the $\mathrm{R}-\mathrm{W}$ model (1) can itself be viewed as a generalization of the Burgers equation (8).

\subsection{Optimal Control and the Logarithmic Transformation for a Simple Diffusion Process}

In [FS] a separate chapter is devoted to the logarithmic transformation in the context of optimal control of stochastic processes. As a basic illustration, which is directly relevant for the rest of this paper, we consider the controlled diffusion process

$$
d X(s)=u(s) d s+\sqrt{D} d W_{s}, \quad t \leq s \leq \tau,
$$


where $u(s)$ is the control variable. We now define a Lagrangian function in the form

$$
\mathcal{L}_{D}(u)=\frac{1}{2 D} u^{2}
$$

and consider:

Optimal Control Problem $\mathcal{P}_{1}$. Determine an optimal control $u^{*}$ which minimizes the cost functional

$$
J(t, x ; u):=E_{t, x} \int_{t}^{\tau} \mathcal{L}_{D}(u(s)) d s+\psi(\tau, X(\tau)),
$$

with $E_{t, x}$ being the conditional expectation starting with the initial conditions $(t, x)$ and a final cost function $\psi(\tau, X(\tau))$.

Writing the optimal cost as

$$
V(t, x)=\inf _{u}\{J(t, x ; u)\},
$$

the dynamic programming equation for $V(t, x)$ reads [FS]

$$
\frac{\partial}{\partial t} V(t, x)+\frac{D}{2} \frac{\partial^{2}}{\partial x^{2}} V(t, x)-\frac{D}{2}\left[\frac{\partial}{\partial x} V(t, x)\right]^{2}=0,
$$

with $V(\tau, x)=\psi(\tau, x)$. Differentiating (18) with respect to $x$ and writing $\hat{h}(x, t)=$ $D(\partial / \partial x) V(t, x)$, we obtain

$$
\frac{\partial}{\partial t} \hat{h}(x, t)+\frac{D}{2} \frac{\partial^{2}}{\partial x^{2}} \hat{h}(x, t)-\hat{h}(x, t) \frac{\partial}{\partial x} \hat{h}(x, t)=0, \quad 0 \leq t \leq \tau,
$$

which is to be solved with the final boundary data $\hat{h}(x, \tau)=D(\partial / \partial x) \psi(\tau, x)$. Equation (19) is the (backward in time) Burgers equation $(t \mapsto-t$ in (8)). Introducing the logarithmic transformation $V(t, x)=-\ln \hat{\varphi}(x, t)$ or equivalently $\hat{h}(x, t)=-D(\partial / \partial x)$ $\ln \hat{\varphi}(x, t),(19)$ is linearized in the form

$$
\frac{\partial}{\partial t} \hat{\varphi}(x, t)+\frac{D}{2} \frac{\partial^{2}}{\partial x^{2}} \hat{\varphi}(x, t)=0,
$$

which is the (backward) heat equation. In the next section the optimal control problem $\mathcal{P}_{1}$ is studied when the underlying dynamics involve a non-diffusive process.

\section{Optimal Control of Piecewise Deterministic Random Evolution Processes}

Now we consider, instead of (14), the random evolution given by

$$
\frac{d}{d s} X(s)=I(i(s)), \quad X(s=t)=x \in \mathbb{R}, \quad t \leq s \leq \tau,
$$


where $i(s) \in\{-1,+1\}$ is a Markovian alternating renewal process [R] with $I(-1)=a$ and $I(+1)=b$. The holding times in states $a$ and $b$ are respectively given by two control variables $u_{+1}(s)$ and $u_{-1}(s)$. We write $\vec{u}(s)=\left(u_{-1}(s), u_{+1}(s)\right) \in[0, \infty) \times[0, \infty)$. Accordingly, the sojourn time in the states $\{a\}$ and $\{b\}$ are exponentially distributed random variables with parameters $u_{+1}(s)$ and $u_{-1}(s)$ respectively.

Consider now the optimal control problem $\mathcal{P}_{2}$ defined by:

Optimal Control Problem $\mathcal{P}_{2}$. Minimize the functional

$$
\begin{aligned}
J(x, t, i ; \vec{u}):=E & \left\{\int_{t}^{\tau} \mathcal{L}(i(s), \vec{u}(s)) d s\right. \\
& +\psi(i(\tau), X(\tau)) \mid i(t)=i, X(t)=x\},
\end{aligned}
$$

where $\psi(i(\tau), x(\tau))$ stands for a final cost at time $\tau$ and the Lagrangian in (22) has the form

$$
\mathcal{L}(i, \vec{u})=u_{i} \ln u_{i}-u_{i}+1=L\left(u_{i}\right), \quad i \in\{-1,+1\} .
$$

Let us define the value functions:

$$
\begin{aligned}
v(x, t, i)=\inf _{\vec{u}(s)} E & \left\{\int_{t}^{\tau}[\mathcal{L}(i(s), \vec{u}(s)) d s\right. \\
& +\psi(i(\tau), X(\tau)) \mid i(t)=i, X(t)=x]\} .
\end{aligned}
$$

In view of the (22), (23) and (24), the associated dynamic programming equations read as

$$
\begin{aligned}
& \frac{\partial}{\partial t} v_{-}(x, t)+I(-1) \frac{\partial}{\partial x} v_{-}(x, t)+\inf _{u_{-1}}\left\{u_{-1}\left[v_{+}(x, t)-v_{-}(x, t)\right]+L\left(u_{-1}\right)\right\}=0 \\
& \frac{\partial}{\partial t} v_{+}(x, t)+I(+1) \frac{\partial}{\partial x} v_{+}(x, t)+\inf _{u_{+1}}\left\{u_{+1}\left[v_{-}(x, t)-v_{+}(x, t)\right]+L\left(u_{+1}\right)\right\}=0
\end{aligned}
$$

with $t<\tau, x \in \mathbb{R}$.

With the (26) and (25), we can now prove the result:

Proposition 1. The dynamic programming equations associated with the optimal control problem $\mathcal{P}_{2}$ are

$$
\frac{\partial}{\partial t} v_{-}(t, x)+a \frac{\partial}{\partial x} v_{-}(t, x)-\exp \left\{v_{-}(t, x)-v_{+}(t, x)\right\}+1=0
$$

and

$$
\frac{\partial}{\partial t} v_{+}(t, x)+b \frac{\partial}{\partial x} v_{+}(t, x)-\exp \left\{v_{+}(t, x)-v_{-}(t, x)\right\}+1=0,
$$

for $t \leq \tau$ and with final boundary data

$$
v_{+}(\tau, x)=\psi_{+}(\tau, x) \quad \text { and } \quad v_{-}(\tau, x)=\psi_{-}(\tau, x)
$$


Furthermore, the optimal control functions are

$$
u_{i(s)}^{*}(s)=\exp \left\{i(s)\left[v_{+}(s, X(s))-v_{-}(s, X(s))\right]\right\} .
$$

Proof of Proposition 1.

$$
\inf _{u}\left\{u\left[v_{ \pm}(x, t)-v_{\mp}(x, t)\right]+L\left(u_{ \pm}\right)\right\}=-\exp \left\{v_{\mp}(x, t)-v_{ \pm}(x, t)\right\}+1 .
$$

It is worth remarking from (29) that $u_{+1}(s, X(s)) \cdot u_{-1}(s, X(s)) \equiv 1$.

Proposition 2. The system (27) and (28) can be linearized by a logarithmic transformation of the type

$$
v_{-}(t, x)=-\ln \varphi_{a}(t, x) \quad \text { and } \quad v_{+}(t, x)=-\ln \varphi_{b}(t, x) .
$$

With this transformation (27) and (28) become

$$
\frac{\partial}{\partial t} \varphi_{a}(t, x)+a \frac{\partial}{\partial x} \varphi_{a}(t, x)-\varphi_{a}(t, x)+\varphi_{b}(t, x)=0
$$

and

$$
\frac{\partial}{\partial t} \varphi_{b}(t, x)+b \frac{\partial}{\partial x} \varphi_{b}(t, x)-\varphi_{b}(t, x)+\varphi_{a}(t, x)=0 .
$$

The boundary conditions are

$$
\varphi_{a}(\tau, x)=e^{-\psi_{-}(\tau, x)}, \quad \varphi_{b}(\tau, x)=e^{-\psi_{+}(\tau, x)} .
$$

Proof of Proposition 2. The proof follows from direct calculations.

Note that according to the general theory developed in [FS], the system (31) and (32) describes the backward Chapman-Kolmogorov equation for the random evolution given by (21) when the transition rates are $u_{-1}(s)=u_{+1}(s) \equiv 1$.

Proposition 3. Equations (27) and (28) are equivalent to the $R$ - $W$ model given in (1).

Proof of Proposition 3. We introduce the new variables

$$
\xi=\frac{1}{b-a}(x-a t) \quad \text { and } \quad \eta=\frac{1}{b-a}(b t-x) .
$$

With these new variables, (27) and (28) read

$$
\frac{\partial}{\partial \eta} v_{-}(\xi, \eta)=\exp \left\{v_{-}(\xi, \eta)-v_{+}(\xi, \eta)\right\}-1
$$

and

$$
\frac{\partial}{\partial \xi} v_{+}(\xi, \eta)=\exp \left\{v_{+}(\xi, \eta)-v_{-}(\xi, \eta)\right\}-1 .
$$


The last equation can be rewritten as

$$
\frac{\partial}{\partial \eta} v_{-}(\xi, \eta)=\exp \left\{v_{-}(\xi, \eta)-v_{+}(\xi, \eta)\right\}\left[1-\exp \left\{v_{+}(\xi, \eta)-v_{-}(\xi, \eta)\right\}\right]
$$

and hence

$$
\frac{\partial}{\partial \eta} v_{-}(\xi, \eta)=\exp \left\{v_{-}(\xi, \eta)-v_{+}(\xi, \eta)\right\}\left[-\frac{\partial}{\partial \xi} v_{+}(\xi, \eta)\right]
$$

This directly implies that

$$
\frac{\partial^{2}}{\partial \xi \partial \eta} v_{+}(\xi, \eta)=\frac{\partial}{\partial \xi} v_{+}(\xi, \eta)+\frac{\partial}{\partial \eta} v_{+}(\xi, \eta)+\frac{\partial}{\partial \xi} v_{+}(\xi, \eta) \frac{\partial}{\partial \eta} v_{+}(\xi, \eta) \text {. }
$$

By writing

$$
2 g(\xi, \eta):=-\frac{\partial}{\partial \xi} v_{+}(\xi, \eta) \quad \text { and } \quad 2 f(\xi, \eta):=\frac{\partial}{\partial \eta} v_{+}(\xi, \eta)
$$

we end with

$$
\frac{\partial}{\partial \xi} f(\xi, \eta)=f(\xi, \eta)-g(\xi, \eta)-2 f(\xi, \eta) g(\xi, \eta)
$$

and

$$
\frac{\partial}{\partial \eta} g(\xi, \eta)=-f(\xi, \eta)+g(\xi, \eta)+2 f(\xi, \eta) g(\xi, \eta) .
$$

In terms of the original variables $(t, x)$, these last equations read

$$
\frac{\partial}{\partial t} f(x, t)+b \frac{\partial}{\partial x} f(x, t)=-[2 f(x, t) g(x, t)-f(x, t)+g(x, t)]
$$

and

$$
\frac{\partial}{\partial t} g(x, t)+a \frac{\partial}{\partial x} g(x, t)=+[2 f(x, t) g(x, t)-f(x, t)+g(x, t)] .
$$

With $a=-b=1$, this is indeed the (backward) R-W model given in (1), if we rescale $x \mapsto \sqrt{D \alpha} x, t \mapsto-2 \alpha t$.

\section{Conclusion and Perspectives}

The study of the relations between non-linear field equations describing non-equilibrium transport phenomena and the logarithmic transformation of the dynamic programming 
equation describing the optimal control and stochastic processes remain, so far, confined to cases for which the underlying dynamics is diffusive. This specific connection is explicitly studied in the contribution of Pavon [Pa] (see also several references therein). Along the same lines, we explore, in the present paper, the possibilities offered by the optimal control of continuous time, piecewise deterministic evolutions. It is shown here how the discrete velocity model of the Boltzmann equation, introduced 20 years ago by Ruijgrok and $\mathrm{Wu}[\mathrm{RW}]$, can be directly related to the control of a simple piecewise deterministic process involving two states. This observation gives additional information helpful to answer the difficult questions raised in [RW], namely: Is the two velocity R-W model of the Boltzmann equation unique? Would it be possible to construct more general models involving three or more discrete velocities?

\section{References}

[AS] M. A Abramovitz and I. A. Stegun. Handbook of Mathematical functions. Dover, New York, 1965

[F] W. H Fleming. Exit probabilities and optimal stochastic control. Appl. Math. Optim. 4 (1978), 329-346.

[FS] W. H Fleming and H. M. Soner. Controlled Markov Processes and Viscosity Solutions. Springer-Verlag, Berlin, 1993. See in particular Chapter 6.

[Hol] C. J. Holland. A new energy characterization of the smallest eigenvalue of the Schroedinger equation. Comm. Pure Appl. Math. 30 (1977), 755-765.

[HS] M.-O. Hongler and L. Streit. A probabilistic connection between the Burgers and a discrete velocity Boltzmann equation. Europhys. Lett. 12 (1990), 193-197.

[Hop] E. Hopf. The partial differential equation $u_{t}+u u_{x}=\mu u_{x x}$. Comm. Pure Appl. Math. 3 (1950), 201-230.

[ML] E. W. Montroll and J. L. Lebowitz. Studies in Statistical Mechanics. North-Holland, Amsterdam, 1979. See Chapter 2.

[Pa] M. Pavon. Stochastic control and non-linear thermodynamical systems. Appl. Math. Optim. 19 (1989), 187-202

[Pi] M. A. Pinsky. Lectures on Random Evolution. World Scientific, Singapore, 1991.

[R] S. M. Ross. Stochastic Processes. Wiley, New York 1983. See Chapter 3.4.1.

[R] Th. W. Ruijgrok and T. T. Wu. A completely solvable model of the non-linear Boltzmann equation. Phys. A 113 (1982), 401-416. 